\title{
Are immune disorders related to quality of diet, physical activity, screen-time and sleep-time in our children?
}

\author{
M. Calvo-Terrades ${ }^{1,2}$ and J. C. Sevilla-Moya ${ }^{2,3}$ \\ ${ }^{1}$ Albera Salut, ABS Peralada (Girona), ${ }^{2}$ Institut Recerca Biomèdica Girona and ${ }^{3}$ Fundació Salut Empordà, \\ Figueres (Girona)
}

Scientific evidence shows increased prevalence and incidence of immune disorders that can be related to changes in life style successful in the last few years.

The purposes of this study were: (1) to know the quality of our children's diet and food groups mean daily portions. (2) To know the intensity and type of physical activity (PhyA) currently performed by our children; the time of sedentary 'screen-time' activities; and sleep time. (3) To determine if significant differences exist in the variables analysed between children with and without chronic diseases. (4) To determine if significant correlations exist between the different variables analysed.

We enrolled 299 children attending from school and primary care centre in our village (rural area). Parent's consent was obtained. Age ranged from 4 to 13 years. We measured height, weight and body mass index (BMI). Diet information was obtained from a 7-day diet questionnaire, the Healthy Nutrition K13Plus (HNK13P) scale, and the Mediterranean Diet in Childhood (MD) scale. PhyA, screen-time and sleep time were obtained from a 7-day questionnaire, and the K13Plus PhyA and 'screen-time' behaviour scale (K13Phy). Pathological history was made emphasising immune diseases.

Immune diseases were observed in 34 children: asthma (12), rhino conjunctivitis (6), pharmacological allergies (7), food allergies (4), diabetes (2), celiac disease (2) and ulcerative colitis (1).

Statistics results

\begin{tabular}{lrr}
\hline & Mean & SD \\
\hline Age & 7.3 & 2.6 \\
HNK13P & 6.9 & 1.8 \\
DM & 7.3 & 1.9 \\
K13Phy & 5.3 & 1.7 \\
BMI & 17.3 & 2.9 \\
\hline
\end{tabular}

\begin{tabular}{lcr}
\hline & Mean & SD \\
\hline Sleep time (h/d) & 10.2 & 4.5 \\
Screen-time (min/d) & 64.6 & 42.5 \\
Low-intensity PhyA (min/d) & 34.3 & 32.1 \\
Moderate-intensity PhyA (min/d) & 54.2 & 49.0 \\
High-intensity PhyA (min/d) & 25.4 & 20.0 \\
\hline
\end{tabular}

\begin{tabular}{|c|c|c|c|c|c|c|c|}
\hline Mean daily portions & Dairy products & Vegetables & Fruits & Protein foods & Carbohydrate & Pastries & $\%$ Vegetable protein intake \\
\hline Observed & 1.6 & 1.1 & 1.1 & 2.4 & 3 & 1.4 & 5.90 \\
\hline Recommended & $2-3$ & $2-3$ & $2-3$ & $2-3$ & $3-6$ & $<0.4$ & 50 \\
\hline
\end{tabular}

No significant correlation was found between absence/presence immune diseases and variables analysed. BMI shows an inverse significant correlation with moderate intensity exercise. We found no significant mean differences between children with and without chronic diseases in the variables analysed.

Conclusions: Our children's eating behaviours are suboptimal. Consumption of dairy products, fruits and vegetables should be increased; pastry and protein of animal origin should be reduced. According to the current recommendations for PhyA in childhood, our results indicate that especially low intensity PhyA should be encouraged. In the prevention and treatment of infantile overweight and/or obesity the relationship between BMI and moderate PhyA must be considered. Control measures for 'screen-time' are required. Life style indicators evaluated in our study did not show any difference between children with and without immunitary diseases, but we must emphasise that the prevalence of these disorders is less frequent in our area than in others geographic areas. 